\title{
On a problem of Steve Kalikow
}

\author{
by
}

Saharon Shelah (Jerusalem and New Brunswick, NJ)

\begin{abstract}
The Kalikow problem for a pair $(\lambda, \kappa)$ of cardinal numbers, $\lambda>\kappa$ (in particular $\kappa=2$ ) is whether we can map the family of $\omega$-sequences from $\lambda$ to the family of $\omega$-sequences from $\kappa$ in a very continuous manner. Namely, we demand that for $\eta, \nu \in{ }^{\omega} \lambda$ we have: $\eta, \nu$ are almost equal if and only if their images are.

We show consistency of the negative answer, e.g., for $\aleph_{\omega}$ but we prove it for smaller cardinals. We indicate a close connection with the free subset property and its variants.
\end{abstract}

0. Introduction. In the present paper we are interested in the following property of pairs of cardinal numbers:

Definition 0.1. Let $\lambda, \kappa$ be cardinals. We say that the pair $(\lambda, \kappa)$ has the Kalikow property (and then we write $\mathcal{K} \mathcal{L}(\lambda, \kappa)$ ) if there is a sequence $\left\langle F_{n}: n<\omega\right\rangle$ of functions such that

$$
F_{n}:{ }^{n} \lambda \rightarrow \kappa \quad(\text { for } n<\omega)
$$

and if $F:{ }^{\omega} \lambda \rightarrow{ }^{\omega} \kappa$ is given by

$$
\left(\forall \eta \in{ }^{\omega} \lambda\right)(\forall n \in \omega)\left(F(\eta)(n)=F_{n}(\eta\lceil n))\right.
$$

then for every $\eta, \nu \in{ }^{\omega} \lambda$,

$$
\left(\forall^{\infty} n\right)(\eta(n)=\nu(n)) \quad \text { iff } \quad\left(\forall^{\infty} n\right)(F(\eta)(n)=F(\nu)(n)) .
$$

In particular we answer the following question of Kalikow:

Kalikow Problem 0.2. Is $\mathcal{K} \mathcal{L}\left(2^{\aleph_{0}}, 2\right)$ provable in ZFC?

The Kalikow property of pairs of cardinals was studied in [Ka90]. Several results are known already. Let us mention some of them. First, one can easily notice that

$$
\mathcal{K} \mathcal{L}(\lambda, \kappa) \& \lambda^{\prime} \leq \lambda \& \kappa^{\prime} \geq \kappa \Rightarrow \mathcal{K} \mathcal{L}\left(\lambda^{\prime}, \kappa^{\prime}\right) .
$$

2000 Mathematics Subject Classification: 03E35, 03E05.

Key words and phrases: set theory, forcing, continuity, Kalikow, free subset.

The research was partially supported by the Israel Science Foundation. Publication 590. 
Also ("transitivity")

$$
\mathcal{K} \mathcal{L}\left(\lambda_{2}, \lambda_{1}\right) \& \mathcal{K} \mathcal{L}\left(\lambda_{1}, \lambda_{0}\right) \Rightarrow \mathcal{K} \mathcal{L}\left(\lambda_{2}, \lambda_{0}\right)
$$

and

$$
\mathcal{K} \mathcal{L}(\lambda, \kappa) \Rightarrow \lambda \leq \kappa^{\aleph_{0}} .
$$

Kalikow proved that $\mathrm{CH}$ implies $\mathcal{K} \mathcal{L}\left(2^{\aleph_{0}}, 2\right)$ (in fact that $\mathcal{K} \mathcal{L}\left(\aleph_{1}, 2\right)$ holds true) and he conjectured that $\mathrm{CH}$ is equivalent to $\mathcal{K} \mathcal{L}\left(2^{\aleph_{0}}, 2\right)$.

The question 0.2 is formulated in [Mi91, Problem 15.15, p. 653].

We shall prove that $\mathcal{K} \mathcal{L}(\lambda, 2)$ is closely tied with some variants of the free subset property (both positively and negatively). First we present an answer to problem 0.2 proving the consistency of $\neg \mathcal{K} \mathcal{L}\left(2^{\aleph_{0}}, 2\right)$ in 1.1 (see 2.8 too). Later we discuss variants of the proof (concerning the cardinal and the forcing). Then we deal with a positive answer, in particular $\mathcal{K} \mathcal{L}\left(\aleph_{n}, 2\right)$, and we show that the negation of a relative of the free subset property for $\lambda$ implies $\mathcal{K} \mathcal{L}(\lambda, 2)$.

We thank the participants of the Jerusalem Logic Seminar 1994/95 and particularly Andrzej Rosłanowski for writing it up so nicely.

Notation. We will use the Greek letters $\kappa, \lambda, \chi$ to denote (infinite) cardinals and the letters $\alpha, \beta, \gamma, \zeta, \xi$ to denote ordinals. Sequences of ordinals will be called $\bar{\alpha}, \bar{\beta}, \bar{\zeta}$ with the usual convention that $\bar{\alpha}=\left\langle\alpha_{n}\right.$ : $n<\lg (\bar{\alpha})\rangle$ etc. Sets of ordinals will be denoted by $u, v, w$ (with possible indexes).

The quantifiers $\left(\forall^{\infty} n\right)$ and $\left(\exists^{\infty} n\right)$ are abbreviations for "for all but finitely many $n \in \omega$ " and "for infinitely many $n \in \omega$ ", respectively.

1. The negative result. For a cardinal $\chi$, the forcing notion $\mathbb{C}_{\chi}$ for adding $\chi$ many Cohen reals consists of finite functions $p$ such that for some $w \in[\chi]^{<\omega}, n<\omega$,

$$
\operatorname{dom}(p)=\{(\zeta, k): \zeta \in w \& k<n\} \quad \text { and } \quad \operatorname{rang}(p) \subseteq 2
$$

ordered by inclusion.

Theorem 1.1. Assume $\lambda \rightarrow\left(\omega_{1} \cdot \omega\right)_{2^{\kappa}}^{<\omega}, 2^{\kappa}<\lambda \leq \chi$. Then

$$
\Vdash_{\mathbb{C}_{\chi}} \neg \mathcal{K} \mathcal{L}(\lambda, \kappa) \quad \text { and hence } \quad \Vdash_{\mathbb{C}_{\chi}} \neg \mathcal{K} \mathcal{L}\left(2^{\aleph_{0}}, 2\right) .
$$

Proof. Suppose that $\mathbb{C}_{\chi}$-names $\underset{\sim}{F_{n}}$ (for $\left.n \in \omega\right)$ and a condition $p \in \mathbb{C}_{\chi}$ are such that

$$
p \Vdash_{\mathbb{C}_{\chi}} "\langle\underset{\sim}{F}: n<\omega\rangle \text { exemplifies } \mathcal{K} \mathcal{L}(\lambda, \kappa) " .
$$

For $\bar{\alpha} \in{ }^{n} \lambda$ choose a maximal antichain $\left\langle p_{\bar{\alpha}, l}^{n}: l<\omega\right\rangle$ of $\mathbb{C}_{\chi}$ deciding the values of $\underset{\sim}{F}(\bar{\alpha})$. Thus we have a sequence $\left\langle\gamma_{\bar{\alpha}, l}^{n}: l<\omega\right\rangle \subseteq \kappa$ such that

$$
p_{\bar{\alpha}, l}^{n} \Vdash_{\mathbb{C}_{\chi}} \underset{\sim}{F}(\bar{\alpha})=\gamma_{\bar{\alpha}, l}^{n} .
$$


Let $\chi^{*}$ be a sufficiently large regular cardinal. Take an elementary submodel $M$ of $\left(\mathcal{H}\left(\chi^{*}\right), \in,<_{\chi^{*}}^{*}\right)$ such that

- $\|M\|=\chi, \chi+1 \subseteq M$,

- $\left\langle p_{\bar{\alpha}, l}^{n}: l<\omega, n \in \omega, \bar{\alpha} \in{ }^{n} \lambda\right\rangle,\left\langle\gamma_{\bar{\alpha}, l}^{n}: l<\omega, n \in \omega, \bar{\alpha} \in{ }^{n} \lambda\right\rangle \in M$.

By $\lambda \rightarrow\left(\omega_{1} \cdot \omega\right)_{2^{\kappa}}^{<\omega}$ (see [Sh 481, Claim 1.3]), we find a set $B \subseteq \lambda$ of indiscernibles in $M$ over

$$
\kappa \cup\left\{\left\langle p_{\bar{\alpha}, l}^{n}: l<\omega: n \in \omega, \bar{\alpha} \in{ }^{n} \lambda\right\rangle,\left\langle\gamma_{\bar{\alpha}, l}^{n}: l<\omega: n \in \omega, \bar{\alpha} \in{ }^{n} \lambda\right\rangle, \chi, p\right\}
$$

and a system $\left\langle N_{u}: u \in[B]^{<\omega}\right\rangle$ of elementary submodels of $M$ such that

(a) $B$ is of order type $\omega_{1} \cdot \omega$ and for $u, v \in[B]^{<\omega}$ :

(b) $\kappa+1 \subseteq N_{u}$,

(c) $\chi, p,\left\langle p_{\bar{\alpha}, l}^{n}: l<\omega, n<\omega, \bar{\alpha} \in{ }^{n} \lambda\right\rangle,\left\langle\gamma_{\bar{\alpha}, l}^{n}: l<\omega, n<\omega, \bar{\alpha} \in{ }^{n} \lambda\right\rangle \in N_{u}$,

(d) $\left|N_{u}\right|=\kappa, N_{u} \cap B=u$,

(e) $N_{u} \cap N_{v}=N_{u \cap v}$,

(f) $|u|=|v| \Rightarrow N_{u} \cong N_{v}$, and let $\pi_{u, v}: N_{v} \rightarrow N_{u}$ be this (unique) isomorphism,

(g) $\pi_{v, v}=\operatorname{id}_{N_{v}}, \pi_{u, v}(v)=u, \pi_{u_{0}, u_{1}} \circ \pi_{u_{1}, u_{2}}=\pi_{u_{0}, u_{2}}$,

(h) if $v^{\prime} \subseteq v,|v|=|u|$ and $u^{\prime}=\pi_{u, v}\left(v^{\prime}\right)$ then $\pi_{u^{\prime}, v^{\prime}} \subseteq \pi_{u, v}$.

Note that if $u \subseteq B$ is of order type $\omega$ then we may define

$$
N_{u}=\bigcup\left\{N_{v}: v \text { is a finite initial segment of } u\right\} .
$$

Then the models $N_{u}$ (for $u \subseteq B$ of order type $\leq \omega$ ) have the properties (b) - (h) too.

Let $\left\langle\beta_{\zeta}: \zeta<\omega_{1} \cdot \omega\right\rangle$ be the increasing enumeration of $B$. For a set $u \subseteq B$ of order type $\leq \omega$ let $\bar{\beta}^{u}$ be the increasing enumeration of $u\left(\operatorname{sol} \lg \left(\bar{\beta}^{u}\right)=|u|\right)$. Let $u^{*}=\left\{\beta_{\omega_{1} \cdot n}: n<\omega\right\}$. For $k \leq \omega$ and a sequence $\bar{\xi}=\left\langle\xi_{m}: m<k\right\rangle \subseteq \omega_{1}$ we define

$$
u[\bar{\xi}]=\left\{\beta_{\omega_{1} \cdot m+\xi_{m}}: m<k\right\} \cup\left\{\beta_{\omega_{1} \cdot n}: n \in \omega \backslash k\right\} .
$$

Now, working in $\mathbf{V}^{\mathbb{C}_{\chi}}$, we say that a sequence $\bar{\xi}$ is $k$-strange if

(1) $\underset{\sim}{\bar{\xi}}$ is a sequence of countable ordinals greater than $0, \lg (\underset{\mathcal{\xi}}{\bar{\xi}})=k \leq \omega$,

(2) $(\forall m<\omega)\left(\underset{\sim}{F}\left(\bar{\beta}^{u[\bar{\xi}]}\lceil m)=\underset{\sim}{F}{ }_{m}\left(\bar{\beta}^{u^{*}}\lceil m)\right)\right.\right.$.

Claim 1.1.1. In $\mathbf{V}^{\mathbb{C}_{\chi}}$, if $\bar{\xi}^{k}$ are $k$-strange sequences (for $k<\omega$ ) such that $(\forall k<\omega)\left(\tilde{\xi}^{k} \triangleleft{\underset{\sim}{\xi}}^{k+1}\right)$ then the sequence $\underset{\sim}{\bar{\xi}}:=\bigcup_{k<\omega} \bar{\xi}^{k}$ is $\omega$-strange.

Proof. Should be clear (note that in this situation we have $\bar{\beta}^{u[\bar{\xi}]}\lceil m=$ $\bar{\beta}^{u\left[\bar{\xi}^{m}\right]}\lceil m)$.

Claim 1.1.2. $p \Vdash_{\mathbb{C}_{\chi}}$ "there are no $\omega$-strange sequences". 
Proof. Assume not. Then we find a name $\underset{\sim}{\bar{\xi}}=\left\langle\underset{\sim}{\xi_{m}}: m<\omega\right\rangle$ for an $\omega$-sequence and a condition $q \geq p$ such that

$$
q \Vdash_{\mathbb{C}_{\chi}} "(\forall m<\omega)\left(0<\underset{\sim}{\xi_{m}}<\omega_{1} \& \underset{\sim}{\underset{\sim}{F}}\left(\bar{\beta}^{u[\bar{\xi}]} \mid m\right)=\underset{\sim}{\underset{\sim}{F}}\left(\bar{\beta}^{u^{*}}\lceil m)\right) " .\right.
$$

By the choice of $p$ and $\underset{\sim}{F}$ we conclude that

$$
q \Vdash_{\mathbb{C}_{\chi}} "\left(\forall^{\infty} m\right)\left(\bar{\beta}^{u[\bar{\xi}]}(m)=\bar{\beta}^{u^{*}}(m)\right) ",
$$

which contradicts the definition of $\bar{\beta}^{u[\bar{\xi}]}, \bar{\beta}^{u^{*}}$, Definition 0.1 and the fact that

$$
q \Vdash_{\mathbb{C}_{\chi}} "(\forall m<\omega)\left(0<\underset{\sim}{\xi_{m}}<\omega_{1}\right) " .
$$

By 1.1.1, 1.1.2, any inductive attempt to construct (in $\mathbf{V}^{\mathbb{C}_{\chi}}$ ) an $\omega$-strange sequence $\bar{\xi}$ has to fail. Consequently, we find a condition $p^{*} \geq p$, an integer $k<\omega$ and a sequence $\bar{\xi}=\left\langle\xi_{l}: l<k\right\rangle$ such that

$$
p^{*} \Vdash_{\mathbb{C}_{\chi}} \text { " } \bar{\xi} \text { is } k \text {-strange but } \neg\left(\exists \xi<\omega_{1}\right)(\bar{\xi} \frown\langle\xi\rangle \text { is }(k+1) \text {-strange)". }
$$

Then in particular

$$
p^{*} \Vdash_{\mathbb{C}_{\chi}} "(\forall m<\omega)\left(\underset { \sim } { F } \left(\bar{\beta}^{u[\bar{\xi}]}\lceil m)=\underset{\sim}{F}\left(\bar{\beta}^{u^{*}}\lceil m)\right) " .\right.\right.
$$

[It may happen that $k=0$, i.e., $\bar{\xi}=\langle\rangle$.]

For $\xi<\omega_{1}$ let $u_{\xi}=u\left[\bar{\xi}\langle\langle\xi\rangle]\right.$ and $w_{\xi}=u_{\xi} \cup\left(u^{*} \backslash\left\{\omega_{1} \cdot k\right\}\right)$. Thus $w_{0}=u[\bar{\xi}] \cup u^{*}$ and all $w_{\xi}$ have order type $\omega$ and $\pi_{w_{\xi_{1}}, w_{\xi_{2}}}$ is the identity on $N_{w_{\xi} \backslash\left\{\omega_{1} \cdot k+\xi_{2}\right\}}$. Let $q:=p^{*}\left\lceil N_{w_{0}}\right.$ and $q_{\xi}=\pi_{w_{\xi}, w_{0}}(q) \in N_{w_{\xi}}\left(\right.$ so $\left.q_{0}=q\right)$. As the isomorphism $\pi_{w_{\xi}, w_{0}}$ is the identity on $N_{w_{0}} \cap N_{w_{\xi}}=N_{w_{0} \cap w_{\xi}}$ (and by the definition of Cohen forcing), we see that the conditions $q, q_{\xi}$ are compatible. Moreover, as $p^{*} \geq p$ and $p \in N_{\emptyset}$, we find that both $q$ and $q_{\xi}$ are stronger than $p$.

Now fix $\xi_{0} \in\left(0, \omega_{1}\right)$ (e.g. $\left.\xi_{0}=1\right)$ and look at the sequences $\bar{\beta}^{u} \xi_{0}$ and $\bar{\beta}^{u^{*}}$. They are eventually equal and hence

$$
p \Vdash_{\mathbb{C}_{\chi}} "\left(\forall^{\infty} m\right)\left(\underset { \sim } { F } \left(\bar{\beta}^{u_{\xi_{0}}}\lceil m)=\underset{\sim}{F}\left(\bar{\beta}^{u^{*}}\lceil m)\right) " .\right.\right.
$$

So we find $m^{*}<\omega$ and a condition $q_{\xi_{0}}^{\prime} \geq q_{\xi_{0}}, q$ such that

$$
\left(\otimes_{q_{\xi_{0}}^{\prime}}^{\xi_{0}, m^{*}}\right) \quad q_{\xi_{0}}^{\prime} \Vdash_{\mathbb{C}_{\chi}} "\left(\forall m \geq m^{*}\right)\left(\underset { \sim } { \underset { F } { F } } \left(\bar{\beta}^{u_{\xi_{0}}}\lceil m)=\underset{\sim}{F}{ }_{m}\left(\bar{\beta}^{u *}\lceil m)\right) "\right.\right.
$$

and (as we can increase $q_{\xi_{0}}^{\prime}$ )

$\left(\oplus_{q_{\xi_{0}}^{\prime}}^{\xi_{0}, m^{*}}\right) \quad q_{\xi_{0}}^{\prime}$ decides the values of $\underset{\sim}{F}\left(\bar{\beta}^{u_{\xi_{0}}}\lceil m)\right.$ and $\underset{\sim}{\underset{F}{F}}\left(\bar{\beta}^{u^{*}}\lceil m)\right.$ for all $m \leq m^{*}$.

Note that the condition $\left(\otimes_{q_{\xi_{0}}^{\prime}}^{\xi_{0}, m^{*}}\right)$ means that there are NO $m \geq m^{*}, l_{0}, l_{1}$ $<\omega$ with $\gamma_{\bar{\beta}^{u} \xi_{0}\left\lceil m, l_{0}\right.}^{m} \neq \gamma_{\bar{\beta}^{u^{*}}\left\lceil m, l_{1}\right.}^{m}$ and the three conditions $q_{\xi_{0}}^{\prime}, p_{\bar{\beta}^{u} \xi_{0}\left\lceil m, l_{0}\right.}^{m}$ and $p_{\bar{\beta}^{*}{ }^{*} \mid m, l_{1}}^{m}$ have a common upper bound in $\mathbb{C}_{\chi}$ (remember the choice of the $p_{\bar{\alpha}, l}^{n}$ 's and $\gamma_{\bar{\alpha}, l}^{n}$ 's). Similarly, the condition $\left(\oplus_{q_{\xi_{0}}^{\prime}}^{\xi_{0}, m^{*}}\right)$ means there are 
NO $m \leq m^{*}, l_{0}, l_{1}<\omega$ with either $\gamma_{\bar{\beta}^{u} \xi_{0}\left\lceil m, l_{0}\right.}^{m} \neq \gamma_{\bar{\beta}^{u} \xi_{0}\left\lceil m, l_{1}\right.}^{m}$ and both $q_{\xi_{0}}^{\prime}$ and $p_{\bar{\beta}^{u} \xi_{0}\left\lceil m, l_{0}\right.}^{m}$, and $q_{\xi_{0}}^{\prime}$ and $p_{\bar{\beta}^{u} \xi_{0}\left\lceil m, l_{1}\right.}^{m}$ are compatible in $\mathbb{C}_{\chi}$, or $\gamma_{\bar{\beta}^{u^{*}}\left\lceil m, l_{0}\right.}^{m} \neq$ $\gamma_{\bar{\beta}^{u^{*}} \mid m, l_{1}}^{m}$ and both $q_{\xi_{0}}^{\prime}$ and $p_{\bar{\beta}^{u^{*}} \mid m, l_{0}}^{m}$, and $q_{\xi_{0}}^{\prime}$ and $p_{\bar{\beta}^{u^{*}} \mid m, l_{1}}^{m}$ are compatible in $\mathbb{C}_{\chi}$.

Consequently, the condition $q_{\xi_{0}}^{*}:=q_{\xi_{0}}^{\prime}\left\lceil N_{w_{0} \cup w_{\xi_{0}}}\right.$ has both properties $\left(\otimes_{q_{\xi_{0}}^{*}}^{\xi_{0}, m^{*}}\right)$ and $\left(\oplus_{q_{\xi_{0}}^{*}}^{\xi_{0}, m^{*}}\right)$ (and it is stronger than both $q$ and $\left.q_{\xi_{0}}\right)$.

Now, for $0<\xi<\omega_{1}$ let

$$
q_{\xi}^{*}:=\pi_{w_{0} \cup w_{\xi}, w_{0} \cup w_{\xi_{0}}}\left(q_{\xi_{0}}^{*}\right) \in N_{w_{0} \cup w_{\xi}} .
$$

Then (for $\left.\xi \in\left(0, \omega_{1}\right)\right)$ the condition $q_{\xi}^{*}$ is stronger than

$$
\text { both } \quad q=\pi_{w_{0} \cup w_{\xi}, w_{0} \cup w_{\xi_{0}}}(q) \text { and } q_{\xi}=\pi_{w_{0} \cup w_{\xi}, w_{0} \cup w_{\xi_{0}}}\left(q_{\xi_{0}}\right)
$$

and it has the properties $\left(\otimes_{q_{\xi}^{*}}^{\xi, m^{*}}\right)$ and $\left(\oplus_{q_{\xi}^{*}}^{\xi, m^{*}}\right)$. Moreover for all $\xi_{1}, \xi_{2}$ the conditions $q_{\xi_{1}}^{*}, q_{\xi_{2}}^{*}$ are compatible. [Why? By the definition of Cohen forcing, and $\pi_{w_{0} \cup w_{\xi_{2}}, w_{0} \cup w_{\xi_{1}}}\left(q_{\xi_{1}}^{*}\right)=q_{\xi_{2}}^{*}$ (chasing arrows) and $\pi_{w_{0} \cup w_{\xi_{2}}, w_{0} \cup w_{\xi_{1}}}$ is the identity on $N_{w_{0} \cup w_{\xi_{2}}} \cap N_{w_{0} \cup w_{\xi_{1}}}=N_{\left(w_{0} \cup w_{\xi_{2}}\right) \cap\left(w_{0} \cup w_{\xi_{1}}\right)}$ (see clauses (e), (f), (h) above).]

CLAIM 1.1.3. For each $\xi_{1}, \xi_{2} \in\left(0, \omega_{1}\right)$ the condition $q_{\xi_{1}}^{*} \cup q_{\xi_{2}}^{*}$ forces in $\mathbb{C}_{\chi}$ that

$$
(\forall m<\omega)\left(\underset { \sim } { F } \left(\bar{\beta}^{u_{\xi_{1}}}\lceil m)=\underset{\sim}{F}\left(\bar{\beta}^{u \xi_{2}}\lceil m)\right) .\right.\right.
$$

Proof. If $m \geq m^{*}$ then, by $\left(\otimes_{q_{\xi_{1}}^{*}}^{\xi_{1}, m^{*}}\right)$ and $\left(\otimes_{q_{\xi_{2}}^{*}}^{\xi_{2}, m^{*}}\right)$ (passing through $\underset{\sim}{F}\left(\bar{\beta}^{u^{*}}\lceil m)\right)$, we get

$$
q_{\xi_{1}}^{*} \cup q_{\xi_{2}}^{*} \Vdash_{\mathbb{C}_{\chi}} " \underset{\sim}{F}\left(\bar{\beta}^{u_{\xi_{1}}}\lceil m)=\underset{\sim}{F}\left(\bar{\beta}^{u_{\xi_{2}}}\lceil m) .\right.\right.
$$

If $m<m^{*}$ then we use $\left(\oplus_{q_{\xi_{1}}^{*}}^{\xi_{1}, m^{*}}\right)$ and $\left(\oplus_{q_{\xi_{2}}^{*}}^{\xi_{1}, m^{*}}\right)$ and the isomorphism: the

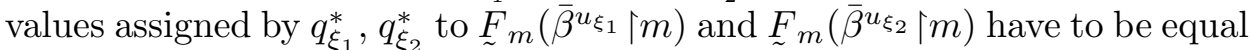
(remember $\kappa \subseteq N_{\emptyset}$, so the isomorphism is the identity on $\kappa$ ).

Look at the conditions

$$
q_{\xi_{1}, \xi_{2}}:=q_{\xi_{1}}^{*}\left\lceil N_{w_{\xi_{1}}} \cup q_{\xi_{2}}^{*} \mid N_{w_{\xi_{2}}} \in N_{w_{\xi_{1}} \cup w_{\xi_{2}}} .\right.
$$

It should be clear that for each $\xi_{1}, \xi_{2} \in\left(0, \omega_{1}\right)$,

$$
q_{\xi_{1}, \xi_{2}} \Vdash_{\mathbb{C}_{\chi}} "(\forall m<\omega)\left(\underset { \sim } { F } \left(\bar{\beta}^{u_{\xi_{1}}}\lceil m)=\underset{\sim}{F}\left(\bar{\beta}^{u} u_{\xi_{2}}\lceil m)\right) " .\right.\right.
$$

Now choose $\xi \in\left(0, \omega_{1}\right)$ so large that

$$
\operatorname{dom}\left(p^{*}\right) \cap\left(N_{w_{\xi}} \backslash N_{w_{0}}\right)=\emptyset
$$

(possible as $\operatorname{dom}\left(p^{*}\right)$ is finite, use (e)). Take any $0<\xi_{1}<\xi_{2}<\omega_{1}$ and put

$$
q^{*}:=\pi_{w_{0} \cup w_{\xi}, w_{\xi_{1}} \cup w_{\xi_{2}}}\left(q_{\xi_{1}, \xi_{2}}\right) .
$$


(Note: $\pi_{w_{0}, w_{\xi_{1}}} \subseteq \pi_{w_{0} \cup w_{\xi}, w_{\xi_{1}} \cup w_{\xi_{2}}}$ and $\left.\pi_{w_{\xi}, w_{\xi_{2}}} \subseteq \pi_{w_{0} \cup w_{\xi}, w_{\xi_{1}} \cup w_{\xi_{2}}}.\right)$ By the isomorphism we get

$$
q^{*} \Vdash_{\mathbb{C}_{\chi}} "(\forall m<\omega)\left(\underset { \sim } { F } \left(\bar{\beta}^{u_{\xi}}\lceil m)=\underset{\sim}{F}\left(\bar{\beta}^{u[\bar{\xi}]}\lceil m)\right) " .\right.\right.
$$

Now look back:

$$
\begin{aligned}
q_{\xi_{1}}^{*} \geq q_{\xi_{1}} & =\pi_{w_{0} \cup w_{\xi_{1}}, w_{0} \cup w_{\xi_{0}}}\left(q_{\xi_{0}}\right)=\pi_{w_{\xi_{1}}, w_{\xi_{0}}}\left(q_{\xi_{0}}\right) \\
& =\pi_{w_{\xi_{1}}, w_{\xi_{0}}}\left(\pi_{w_{\xi_{0}}, w_{0}}(q)\right)=\pi_{w_{\xi_{1}}, w_{0}}(q)
\end{aligned}
$$

and hence

$$
q_{\xi_{1}}^{*}\left\lceil N_{w_{\xi_{1}}} \geq \pi_{w_{\xi_{1}}, w_{0}}(q)\right.
$$

and thus

$$
q^{*}\left\lceil N_{w_{0}} \geq \pi_{w_{0}, w_{\xi_{1}}}\left(q_{\xi_{1}}^{*}\left\lceil N_{w_{\xi_{1}}}\right) \geq q=p^{*}\left\lceil N_{w_{0}} .\right.\right.\right.
$$

Consequently, by the choice of $\xi$, the conditions $q^{*}$ and $p^{*}$ are compatible (remember the definition of $q_{\xi_{1}, \xi_{2}}$ and $q^{*}$ ). Now use $(\otimes)$ to conclude that

$$
q^{*} \cup p^{*} \Vdash_{\mathbb{C}_{\chi}} "(\forall m<\omega)\left(\underset { \sim } { F _ { m } } \left(\bar{\beta}^{u^{*}}\lceil m)=\underset{\sim}{\underset{F}{F}}\left(\bar{\beta}^{u[\bar{\xi}]}\lceil m)=\underset{\sim}{F}\left(\bar{\beta}^{u_{\xi}}\lceil m)\right) ",\right.\right.\right.
$$

which implies that $q^{*} \cup p^{*} \Vdash_{\mathbb{C}_{\chi}}$ " $\bar{\xi}\langle\langle\xi\rangle$ is $(k+1)$-strange", a contradiction.

REMARK 1.2. About the proof of 1.1:

(1) No harm is done by forgetting 0 and replacing it by $\xi_{1}, \xi_{2}$.

(2) A small modification of the proof shows that in $\mathbf{V}^{\mathbb{C}_{\chi}}:$ If $F_{n}:{ }^{n} \lambda \rightarrow \kappa$ $(n \in \omega)$ are such that

$$
\left(\forall \eta, \nu \in{ }^{\omega} \lambda\right)\left[( \forall ^ { \infty } n ) ( \eta ( n ) = \nu ( n ) ) \Rightarrow ( \forall ^ { \infty } n ) \left(F_{n}\left(\eta\lceil n)=F_{n}(\nu\lceil n))\right]\right.\right.
$$

then there are infinite sets $X_{n} \subseteq \lambda($ for $n<\omega)$ such that

$$
(\forall n<\omega)\left(\forall \nu, \eta \in \prod_{l<n} X_{l}\right)\left(F_{n}(\nu)=F_{n}(\eta)\right) .
$$

Say we shall have $X_{n}=\left\{\gamma_{n, i}: i<\omega\right\}$. Starting we have $\gamma_{0}^{*}, \ldots, \gamma_{n}^{*}, \ldots$ In the proof at stage $n$ we have determined $\gamma_{l, i}(l, i<n)$ and $p \in G$, $p \in N_{\left\{\gamma_{l, i}: l, i<\omega\right\} \cup\left\{\gamma_{n}^{*}, \gamma_{n+1}^{*}, \ldots\right\}}$. For $n=0,1,2$ as before. For $n+1>2$ first $\gamma_{0, n}, \ldots, \gamma_{n-1, n}$ are easy by transitivity of equalities. Then find $\gamma_{n, 0}, \gamma_{n, 1}$ as before, and then again duplicate.

(3) In the proof it is enough to use $\left\{\beta_{\omega \cdot n+l}: n<\omega, l<\omega\right\}$. Hence, by 1.2 of [Sh 481] it is enough to assume $\lambda \rightarrow\left(\omega^{3}\right)_{2^{\kappa}}^{<\omega}$. This condition is compatible with $\mathbf{V}=\mathbf{L}$.

(4) We can use only $\lambda \rightarrow\left(\omega^{2}\right)_{2^{\kappa}}^{<\omega}$

Definition 1.3. (1) For a sequence $\bar{\lambda}=\left\langle\lambda_{n}: n<\omega\right\rangle$ of cardinals we define the property $(\circledast)_{\bar{\lambda}}$ :

$(\circledast)_{\bar{\lambda}} \quad$ for every model $M$ of a countable language with universe $\sup _{n \in \omega} \lambda_{n}$ and Skolem functions (for simplicity) there is a sequence $\left\langle X_{n}\right.$ : $n<\omega\rangle$ such that 
(a) $X_{n} \in\left[\lambda_{n}\right]^{\lambda_{n}}$ (actually $X_{n} \in\left[\lambda_{n}\right]^{\omega_{1}}$ suffices)

(b) for every $n<\omega$ and $\bar{\alpha}=\left\langle\alpha_{l}: l \in[n+1, \omega)\right\rangle \in \prod_{l \geq n+1} X_{l}$, letting (for $\xi \in X_{n}$ )

$$
M_{\bar{\alpha}}^{\xi}=\operatorname{Sk}\left(\bigcup_{l<n} X_{l} \cup\{\xi\} \cup\left\{\alpha_{l}: l \in[n+1, \omega)\right\}\right)
$$

we have:

$(\bigoplus)$ the sequence $\left\langle M_{\bar{\alpha}}^{\xi}: \xi \in X_{n}\right\rangle$ forms a $\Delta$-system with heart $N_{\bar{\alpha}}$ and its elements are pairwise isomorphic over the heart $N_{\bar{\alpha}}$.

(2) For a cardinal $\lambda$ the condition $(\circledast)^{\lambda}$ is:

$(\circledast)^{\lambda} \quad$ there exists a sequence $\bar{\lambda}=\left\langle\lambda_{n}: n<\omega\right\rangle$ such that $\sum_{n<\omega} \lambda_{n}=\lambda$ and the condition $(\circledast)_{\bar{\lambda}}$ holds true.

In [Sh 76] a condition $(*)_{\lambda}$, weaker than $(\circledast)^{\lambda}$, was considered. Now, [Sh 124] continues [Sh 76] to get stronger indiscernibility. But by the same proof (using $\omega$-measurable) one can show the consistency of $(\circledast)^{\aleph_{\omega}}+\mathrm{GCH}$.

Note that to carry out the proof of 1.1 we need even less than $(\circledast)^{\lambda}$ : the $\bigcup_{l<n} X_{l}$ (in (b) of 1.3) is much more than needed; it suffices to have $\bar{\beta}^{0} \cup \bar{\beta}^{1}$ where $\bar{\beta}^{0}, \bar{\beta}^{1} \in \prod_{l<n} X_{l}$.

Conclusion 1.4. It is consistent that

$$
2^{\aleph_{0}}=\aleph_{\omega+1} \quad \text { and } \quad \bigwedge_{n<\omega} \neg \mathcal{K} \mathcal{L}\left(\aleph_{\omega}, \aleph_{n}\right) \quad \text { so } \quad \neg \mathcal{K} \mathcal{L}\left(2^{\aleph_{0}}, 2\right) .
$$

REMARK 1.5. Koepke [Ko84] continues [Sh 76] to get equiconsistency. His refinement of [Sh 76] (for the upper bound) works below too.

2. The positive result. For an algebra $M$ on $\lambda$ and a set $X \subseteq \lambda$ the closure of $X$ under functions of $M$ is denoted by $\operatorname{cl}_{M}(X)$. Before proving our result (2.6) we remind the reader of some definitions and propositions.

Proposition 2.1. For an algebra $M$ on $\lambda$ the following conditions are equivalent:

$(\star)_{M}^{0} \quad$ for each sequence $\left\langle\alpha_{n}: n \in \omega\right\rangle \subseteq \lambda$ we have

$$
\left(\forall^{\infty} n\right)\left(\alpha_{n} \in \operatorname{cl}_{M}\left(\left\{\alpha_{k}: n<k<\omega\right\}\right)\right),
$$

$(\star)_{M}^{1} \quad$ there is no sequence $\left\langle A_{n}: n \in \omega\right\rangle \subseteq[\lambda]^{\aleph_{0}}$ such that

$$
(\forall n \in \omega)\left(\operatorname{cl}_{M}\left(A_{n+1}\right) \varsubsetneqq \operatorname{cl}_{M}\left(A_{n}\right)\right),
$$

$(\star)_{M}^{2} \quad\left(\forall A \in[\lambda]^{\aleph_{0}}\right)\left(\exists B \in[A]^{\aleph_{0}}\right)\left(\forall C \in[B]^{\aleph_{0}}\right)\left(\operatorname{cl}_{M}(B)=\operatorname{cl}_{M}(C)\right)$.

Definition 2.2. We say that a cardinal $\lambda$ has the $(\star)$-property for $\kappa$ (and then we write $\operatorname{Pr} \star(\lambda, \kappa))$ if there is an algebra $M$ on $\lambda$ with vocabulary 
of cardinality $\leq \kappa$ satisfying one (equivalently: all) of the conditions $(\star)_{M}^{i}$ $(i<3)$ of 2.1. If $\kappa=\aleph_{0}$ we may omit it.

Remember

Proposition 2.3. If $\mathbf{V}_{0} \subseteq \mathbf{V}_{1}$ are universes of set theory and $\mathbf{V}_{1}=$ $\neg \operatorname{Pr}^{\star}(\lambda)$ then $\mathbf{V}_{0} \models \neg \operatorname{Pr}^{\star}(\lambda)$.

Proof. By absoluteness of the existence of an $\omega$-branch of a tree.

REMARK 2.4. The property $\neg \operatorname{Pr}^{\star}(\lambda)$ is a kind of large cardinal property. It was clarified in $\mathbf{L}$ (remember that it is inherited from $\mathbf{V}$ to $\mathbf{L}$ ) by Silver [Si70] to be equiconsistent with "there is a beautiful cardinal" (terminology of 2.3 of [Sh 110]), another partition property inherited by L. More in [Sh 513].

Proposition 2.5. For each $n \in \omega, \operatorname{Pr}^{\star}\left(\aleph_{n}\right)$.

Proof. This was done in [Sh:b, Chapter XIII], see [Sh:g, Chapter VII] too, and probably earlier by Silver. However, for the sake of completeness we will give the proof.

First note that clearly $\operatorname{Pr}{ }^{\star}\left(\aleph_{0}\right)$ and thus we have to deal with the case when $n>0$. Let $f, g: \aleph_{n} \rightarrow \aleph_{n}$ be two functions such that if $m<n$, $\alpha \in\left[\aleph_{m}, \aleph_{m+1}\right)$ then $f(\alpha, \cdot)\left|\alpha: \alpha \stackrel{1-1}{\rightarrow} \aleph_{m}, g(\alpha, \cdot)\right| \aleph_{m}: \aleph_{m} \stackrel{1-1}{\rightarrow} \alpha$ are functions inverse to each other.

Let $M$ be the following algebra on $\aleph_{n}$ :

$$
M=\left(\aleph_{n}, f, g, m\right)_{m \in \omega} .
$$

We want to check the condition $(\star)_{M}^{1}$ : assume that a sequence $\left\langle A_{k}: k<\omega\right\rangle$ $\subseteq\left[\aleph_{n}\right]^{\aleph_{0}}$ is such that for each $k<\omega$,

$$
\operatorname{cl}_{M}\left(A_{k+1}\right) \varsubsetneqq \operatorname{cl}_{M}\left(A_{k}\right) .
$$

For each $m<n$, the sequence $\left\langle\sup \left(\operatorname{cl}_{M}\left(A_{k}\right) \cap \aleph_{m+1}\right): k<\omega\right\rangle$ is nonincreasing and therefore it is eventually constant. Consequently, we find $k^{*}$ such that

$$
(\forall m<n)\left(\sup \left(\operatorname{cl}_{M}\left(A_{k^{*}+1}\right) \cap \aleph_{m+1}\right)=\sup \left(\operatorname{cl}_{M}\left(A_{k^{*}}\right) \cap \aleph_{m+1}\right)\right) .
$$

By the choice of $\left\langle A_{k}: k<\omega\right\rangle$ we have $\operatorname{cl}_{M}\left(A_{k^{*}+1}\right) \varsubsetneqq \operatorname{cl}_{M}\left(A_{k^{*}}\right)$. Let

$$
\alpha_{0}:=\min \left(\operatorname{cl}_{M}\left(A_{k^{*}}\right) \backslash \mathrm{cl}_{M}\left(A_{k^{*}+1}\right)\right) \text {. }
$$

As the model $M$ contains individual constants $m$ (for $m \in \omega$ ) we know that $\aleph_{0} \subseteq \operatorname{cl}_{M}(\emptyset)$ and hence $\aleph_{0} \leq \alpha_{0}$. Let $m<n$ be such that $\aleph_{m} \leq \alpha_{0}<\aleph_{m+1}$. By the choice of $k^{*}$ we find $\beta \in \operatorname{cl}_{M}\left(A_{k^{*}+1}\right) \cap \aleph_{m+1}$ such that $\alpha_{0} \leq \beta$. Then necessarily $\alpha_{0}<\beta$. Look at $f\left(\beta, \alpha_{0}\right)$ : we know that $\alpha_{0}, \beta \in \mathrm{cl}_{M}\left(A_{k^{*}}\right)$ and therefore $f\left(\beta, \alpha_{0}\right) \in \operatorname{cl}_{M}\left(A_{k^{*}}\right) \cap \aleph_{m}$ and $f\left(\beta, \alpha_{0}\right)<\alpha_{0}$. The minimality of $\alpha_{0}$ implies that $f\left(\beta, \alpha_{0}\right) \in \operatorname{cl}_{M}\left(A_{k^{*}+1}\right)$ and hence

$$
\alpha_{0}=g\left(\beta, f\left(\beta, \alpha_{0}\right)\right) \in \operatorname{cl}_{M}\left(A_{k^{*}+1}\right),
$$

a contradiction. 
Explanation. Better think of the proof below from the end. Let $\bar{\alpha}=$ $\left\langle\alpha_{n}: n\langle\omega\rangle \in{ }^{\omega} \lambda\right.$. So for some $n(*), n(*) \leq n<\omega \Rightarrow \alpha_{n} \in \operatorname{cl}_{M}\left(\alpha_{l}: l>n\right)$. So for some $m_{n}>n,\left\{\alpha_{n(*)}, \ldots, \alpha_{n-1}\right\} \subseteq \operatorname{cl}_{M}\left(\alpha_{n}, \ldots, \alpha_{m_{m}-1}\right)$ and

$$
(\forall l<n(*))\left(\alpha_{l} \in \mathrm{cl}_{M}\left(\alpha_{k}: k>n(*)\right) \quad \Rightarrow \quad \alpha_{l} \in \operatorname{cl}_{M}\left(\alpha_{k}: k \in\left[n, m_{n}\right)\right)\right) .
$$

Let $w=\left\{l<n(*): \alpha_{l} \in \operatorname{cl}_{M}\left(\alpha_{n}: n \geq n(*)\right)\right.$. It is natural to aim at:

(*) for $n$ large enough (say $\left.n>m_{n(*)}\right), F_{n}\left(\left\langle\alpha_{l}: l<n\right\rangle\right)$ depends just on $\left\{\alpha_{l}: l \in[n(*), n)\right.$ or $\left.l \in w\right\}$ and $\left\langle F_{m}(\bar{\alpha} \mid m): m \geq n\right\rangle$ codes $\bar{\alpha} \uparrow(w \cup[n(*), \omega))$.

Of course, we are given an $n$ and we do not know how to compute the real $n(*)$, but we can approximate. Then we look at a late enough end segment where we compute down.

Theorem 2.6. Assume that $\lambda \leq 2^{\aleph_{0}}$ is such that $\operatorname{Pr}^{\star}(\lambda)$ holds. Then $\mathcal{K} \mathcal{L}(\lambda, \omega)$ (and hence $\mathcal{K} \mathcal{L}(\lambda, 2)$ ).

Pr o o f. We have to construct functions $F_{n}:{ }^{n} \lambda \rightarrow \omega$ witnessing $\mathcal{K} \mathcal{L}(\lambda, \omega)$. For this we will introduce functions $\mathbf{k}$ and $\mathbf{l}$ such that for $\bar{\alpha} \in{ }^{n} \lambda$ the value of $\mathbf{k}(\bar{\alpha})$ will say which initial segment of $\bar{\alpha}$ will be irrelevant for $F_{n}(\bar{\alpha})$ and $\mathbf{l}(\bar{\alpha})$ will be such that (under certain circumstances) elements $\alpha_{i}$ (for $\mathbf{k}(\bar{\alpha}) \leq i<\mathbf{l}(\bar{\alpha}))$ will be encoded by $\left\langle\alpha_{j}: j \in[\mathbf{l}(\bar{\alpha}), n)\right\rangle$.

Fix a sequence $\left\langle\eta_{\alpha}: \alpha<\lambda\right\rangle \subseteq{ }^{\omega} 2$ with no repetitions.

Let $M$ be an algebra on $\lambda$ such that $(\star)_{M}^{0}$ holds true. We may assume that there are no individual constants in $M\left(\operatorname{socl}_{M}(\emptyset)=\emptyset\right)$.

Let $\left\langle\tau_{l}^{n}\left(x_{0}, \ldots, x_{n-1}\right): l<\omega\right\rangle$ list all $n$-place terms of the language of the algebra $M$ (and $\tau_{0}^{1}(x)$ is $x$ ) when $0<n<\omega$. For $\bar{\alpha} \in{ }^{\omega \geq} \lambda$ (with $\alpha_{j}$ the $j$ th element in $\bar{\alpha})$ let

$$
u(\bar{\alpha})=\left\{l<\lg (\bar{\alpha}): \alpha_{l} \notin \operatorname{cl}_{M}(\bar{\alpha} \uparrow(l, \lg (\bar{\alpha})))\right\} \cup\{0\}
$$

and for $l \notin u(\bar{\alpha}), l<\lg (\bar{\alpha})$ let

$$
\begin{aligned}
& f_{l}(\bar{\alpha})=\min \left\{j: \alpha_{l} \in \operatorname{cl}_{M}(\bar{\alpha} \uparrow(l, j))\right\}, \\
& g_{l}(\bar{\alpha})=\min \left\{i: \alpha_{l}=\tau_{i}^{f_{l}(\bar{\alpha})-l-1}\left(\bar{\alpha} \uparrow\left(l, f_{l}(\bar{\alpha})\right)\right)\right\} .
\end{aligned}
$$

For $\bar{\alpha} \in{ }^{n} \lambda(1<n<\omega)$ put

$$
\begin{aligned}
& k_{1}(\bar{\alpha})=\min ((u(\bar{\alpha} \uparrow(n-1)) \backslash u(\bar{\alpha})) \cup\{n-1\}), \\
& k_{0}(\bar{\alpha})=\max \left(u(\bar{\alpha}) \cap k_{1}(\bar{\alpha})\right) .
\end{aligned}
$$

Note that if ( $n>1$ and) $\bar{\alpha} \in{ }^{n} \lambda$ then $n-1 \in u(\bar{\alpha})\left(\operatorname{ascl}_{M}(\emptyset)=\emptyset\right.$ ) and $k_{1}(\bar{\alpha})>0$ (as always $0 \in u(\bar{\beta})$ ) and $k_{0}(\bar{\alpha})$ is well defined (as $0 \in u(\bar{\alpha}) \cap k_{1}(\bar{\alpha})$ ) and $k_{0}(\bar{\alpha})<k_{1}(\bar{\alpha})<n$. Moreover, for all $l \in\left(k_{0}(\bar{\alpha}), k_{1}(\bar{\alpha})\right)$ we have $\alpha_{l} \notin u(\bar{\alpha})$ by the choice of $k_{0}(\bar{\alpha})$, hence $\alpha_{l} \notin u(\bar{\alpha} \uparrow(n-1))$ by the choice of $k_{1}(\bar{\alpha})$ and thus $\alpha_{l} \in \operatorname{cl}_{M}(\bar{\alpha} \uparrow(l, n-1))$. Now, for $\bar{\alpha} \in{ }^{\omega>} \lambda, \lg (\bar{\alpha})>1$ we define

$$
\mathbf{l}(\bar{\alpha})=\max \left\{j \leq k_{1}(\bar{\alpha}): j>k_{0}(\bar{\alpha}) \Rightarrow\left(\forall i \in\left(k_{0}(\bar{\alpha}), j\right)\right)\left(g_{i}(\bar{\alpha}) \leq \lg (\bar{\alpha})\right)\right\},
$$




$$
\begin{aligned}
\mathbf{m}(\bar{\alpha}) & =\max \left\{j \leq \mathbf{l}(\bar{\alpha}): j>\max \left\{1, k_{0}(\bar{\alpha})\right\} \Rightarrow k_{0}\left(\bar{\alpha}\lceil j)=k_{0}(\bar{\alpha})\right\},\right. \\
\mathbf{k}(\bar{\alpha}) & =\mathbf{l}(\bar{\alpha}\lceil\mathbf{m}(\bar{\alpha})) \quad(\text { if } \mathbf{m}(\bar{\alpha}) \leq 1 \text { then put } \mathbf{k}(\bar{\alpha})=-1) .
\end{aligned}
$$

Clearly $\mathbf{k}(\bar{\alpha})<\mathbf{m}(\bar{\alpha}) \leq \mathbf{l}(\bar{\alpha}) \leq k_{1}(\bar{\alpha})<\lg (\bar{\alpha})$.

Claim 2.6.1. For each $\bar{\alpha} \in{ }^{\omega} \lambda$, the set $u(\bar{\alpha})$ is finite and:

(1) The sequence $\left\langle k_{1}(\bar{\alpha}\lceil n): n<\omega\rangle\right.$ diverges to $\infty$.

(2) The sequence $\left\langle k_{0}\left(\bar{\alpha}\lceil n): n<\omega \& k_{0}(\bar{\alpha}\lceil n) \neq \max u(\bar{\alpha})\rangle\right.\right.$, if infinite, diverges to $\infty$. There are infinitely many $n<\omega$ with $k_{0}(\bar{\alpha}\lceil n)=\max u(\bar{\alpha})$.

(3) The sequence $\langle\mathbf{l}(\bar{\alpha}\lceil n): n<\omega\rangle$ diverges to $\infty$.

(4) The sequences $\langle\mathbf{m}(\bar{\alpha}\lceil n): n<\omega\rangle$ and $\langle\mathbf{k}(\bar{\alpha}\lceil n): n<\omega\rangle$ diverge to $\infty$.

Proof. Let $\bar{\alpha}=\left\langle\alpha_{n}: n\langle\omega\rangle \in{ }^{\omega} \lambda\right.$. By the property $(\star)_{M}^{0}$ we find $n^{*}<\omega$ such that $u(\bar{\alpha}) \subseteq n^{*}$. Fix $n_{0}>n^{*}$ and define

$$
n_{1}=\max \left\{f_{n}(\bar{\alpha})+g_{n}(\bar{\alpha})+2: n \in\left(n_{0}+1\right) \backslash u(\bar{\alpha})\right\}
$$

(so as $\mathrm{cl}_{M}(\emptyset)=\emptyset$ we have $n_{1} \geq f_{n_{0}}(\bar{\alpha})+2>n_{0}+3$ and for $l \in\left(n_{0}+1\right) \backslash u(\bar{\alpha})$, $\alpha_{l} \in \operatorname{cl}_{M}\left(\alpha_{l+1}, \ldots, \alpha_{n_{1}-1}\right)$ is witnessed by $\tau_{g_{l}(\bar{\alpha})}^{f_{l}(\bar{x})-l-1}\left(\alpha_{l+1}, \ldots, \alpha_{f_{l}(\bar{\alpha})-1}\right)$ with $\left.f_{l}(\bar{\alpha}), g_{l}(\bar{\alpha})<n_{1}-1\right)$.

(1) Note that $u\left(\bar{\alpha}\lceil n) \cap\left(n_{0}+1\right)=u(\bar{\alpha})\right.$ for all $n \geq n_{1}-1$ and hence for $n \geq n_{1}$,

$$
u\left(\bar{\alpha}\lceil n) \cap\left(n_{0}+1\right)=u\left(\bar{\alpha}\lceil(n-1)) \cap\left(n_{0}+1\right) .\right.\right.
$$

Consequently, for all $n \geq n_{1}$ we have $k_{1}\left(\bar{\alpha}\lceil n)>n_{0}\right.$. As we could have chosen $n_{0}$ arbitrarily large we may conclude that $\lim _{n \rightarrow \infty} k_{1}(\bar{\alpha}\lceil n)=\infty$.

(2) Note that for all $n \geq n_{1}$,

$$
\text { either } \quad k_{0}\left(\overline { \alpha } \lceil n ) = \operatorname { m a x } ( u ( \overline { \alpha } ) ) \quad \text { or } \quad k _ { 0 } \left(\bar{\alpha}\lceil n)>n_{0} .\right.\right.
$$

Hence, by the arbitrariness of $n_{0}$, we get the first part of (2).

Let $l^{*}=\min \left(u\left(\bar{\alpha}\left\lceil n_{1}\right) \backslash u(\bar{\alpha})\right)\right.$ (note that $n_{1}-1 \in u\left(\bar{\alpha}\left\lceil n_{1}\right) \backslash u(\bar{\alpha})\right)$. Clearly $l^{*}>n_{0}$ and $\alpha_{l^{*}} \notin u(\bar{\alpha})$. Consider $n=f_{l^{*}}(\bar{\alpha})$ (so $\left.l^{*} \leq n-2, n_{1} \leq n-1\right)$. Then $l^{*} \in u(\bar{\alpha}\lceil(n-1)) \backslash u(\bar{\alpha}\lceil n)$. As

$$
l^{*} \cap u\left(\bar{\alpha}\left\lceil n_{1}\right)=l^{*} \cap u(\bar{\alpha}\lceil n-1)=u(\bar{\alpha})\right.
$$

(remember the choice of $l^{*}$ ) we conclude that

$$
l^{*}=k_{1}\left(\bar{\alpha}\lceil n) \quad \text { and } \quad k_{0}(\bar{\alpha}\lceil n)=\max u(\bar{\alpha}) .\right.
$$

Now, since $n_{0}$ was arbitrarily large, we find that for infinitely many $n$, $k_{0}(\bar{\alpha}\lceil n)=\max u(\bar{\alpha})$.

(3) Suppose that $n \geq n_{1}$. Then we know that $k_{1}\left(\bar{\alpha}\lceil n)>n_{0}\right.$ and either $k_{0}\left(\bar{\alpha}\lceil n)=\max u(\bar{\alpha})\right.$ or $k_{0}\left(\bar{\alpha}\lceil n)>n_{0}\right.$ (see above). If the first possibility takes place then, as $n \geq n_{1}$, we may use $j=n_{0}+1$ to witness that $\mathbf{l}(\bar{\alpha}\lceil n)>$ $n_{0}$ (remember the choice of $\left.n_{1}\right)$. If $k_{0}\left(\bar{\alpha}\lceil n)>n_{0}\right.$ then clearly $\mathbf{l}\left(\bar{\alpha}\lceil n)>n_{0}\right.$. As $n_{0}$ could be arbitrarily large we are done. 
(4) Suppose we are given $m_{0}<\omega$. Take $m_{1}>m_{0}$ such that for all $n \geq m_{1}$,

$$
\text { either } \quad k_{0}\left(\overline { \alpha } \lceil n ) = \operatorname { m a x } u ( \overline { \alpha } ) \quad \text { or } \quad k _ { 0 } \left(\bar{\alpha}\lceil n)>m_{0}\right.\right.
$$

(possible by (2)) and then choose $m_{2}>m_{1}$ such that $k_{0}\left(\bar{\alpha}\left\lceil m_{2}\right)=\max u(\bar{\alpha})\right.$ (by (2)). Due to (3) we find $m_{3}>m_{2}$ such that for all $n \geq m_{3}, \mathbf{l}\left(\bar{\alpha}\lceil n)>m_{2}\right.$. Now suppose that $n \geq m_{3}$. If $k_{0}\left(\bar{\alpha}\lceil n)=\max u(\bar{\alpha})\right.$ then, as $\mathbf{l}\left(\bar{\alpha}\lceil n)>m_{2}\right.$, we get $\mathbf{m}\left(\bar{\alpha}\lceil n) \geq m_{2}>m_{0}\right.$. Otherwise $k_{0}\left(\bar{\alpha}\lceil n)>m_{0}\right.$ (as $\left.n>m_{1}\right)$ and hence $\mathbf{m}\left(\bar{\alpha}\lceil n)>m_{0}\right.$. This shows that $\lim _{n \rightarrow \infty} \mathbf{m}(\bar{\alpha}\lceil n)=\infty$. Now, immediately by the definition of $\mathbf{k}$ and (3) above we conclude that $\lim _{n \rightarrow \infty} \mathbf{k}(\bar{\alpha}\lceil n)=\infty$.

Claim 2.6.2. If $\bar{\alpha}^{1}, \bar{\alpha}^{2} \in{ }^{\omega} \lambda$ are such that $\left(\forall^{\infty} n\right)\left(\alpha_{n}^{1}=\alpha_{n}^{2}\right)$ then $\left(\forall^{\infty} n\right)\left(\mathbf{l}\left(\bar{\alpha}^{1}\lceil n)=\mathbf{l}\left(\bar{\alpha}^{2}\lceil n) \& \mathbf{m}\left(\bar{\alpha}^{1}\lceil n)=\mathbf{m}\left(\bar{\alpha}^{2}\lceil n) \& \mathbf{k}\left(\bar{\alpha}^{1}\lceil n)=\mathbf{k}\left(\bar{\alpha}^{2}\lceil n)\right)\right.\right.\right.\right.\right.\right.$.

Proof. Let $n_{0}$ be greater than $\max \left(u\left(\bar{\alpha}^{1}\right) \cup u\left(\bar{\alpha}^{2}\right)\right)$ and such that

$$
\bar{\alpha}^{1} \uparrow\left[n_{0}, \omega\right)=\bar{\alpha}^{2} \uparrow\left[n_{0}, \omega\right) .
$$

For $k=1,2,3$ define $n_{k}$ by

$$
n_{k+1}=\max \left\{f_{n}\left(\bar{\alpha}^{i}\right)+g_{n}\left(\bar{\alpha}^{i}\right)+2: n \in\left(n_{k}+1\right) \backslash u\left(\bar{\alpha}^{i}\right), i<2\right\} .
$$

As in the proof of 2.6.1, for $i=1,2$ and $j<3$ we have:

$\left(\otimes^{1}\right) \quad\left(\forall n \geq n_{j+1}\right)\left(k_{0}\left(\bar{\alpha}^{i}\lceil n)=\max u\left(\bar{\alpha}^{i}\right)\right.\right.$ or $k_{0}\left(\bar{\alpha}^{i}\lceil n)>n_{j}\right)$,

$\left(\otimes^{2}\right) \quad\left(\forall n \geq n_{j+1}\right)\left(k_{1}\left(\bar{\alpha}^{i}\lceil n)>n_{j} \& \mathbf{l}\left(\bar{\alpha}^{i}\lceil n)>n_{j} \& \mathbf{m}\left(\bar{\alpha}^{i}\lceil n)>n_{j} \&\right.\right.\right.\right.$ $\mathbf{h}\left(\bar{\alpha}^{i}\lceil n)>n_{j}\right)$,

$\left(\otimes^{3}\right) \quad\left(\exists n^{\prime} \in\left(n_{1}, n_{2}\right)\right)\left(k_{0}\left(\bar{\alpha}^{1}\left\lceil n^{\prime}\right)=\max u\left(\bar{\alpha}^{1}\right) \& k_{0}\left(\bar{\alpha}^{2}\left\lceil n^{\prime}\right)=\max u\left(\bar{\alpha}^{2}\right)\right)\right.\right.$

(for $\left(\otimes^{3}\right)$ repeat arguments from 2.6.1(2) and use the fact that $\bar{\alpha}^{1} \uparrow\left[n_{0}, \omega\right)=$ $\left.\bar{\alpha}^{2} \uparrow\left[n_{0}, \omega\right)\right)$. Clearly

$\left(\otimes^{4}\right) \quad\left(\forall n>n_{0}\right)\left(u\left(\bar{\alpha}^{1}\lceil n) \backslash n_{0}=u\left(\bar{\alpha}^{2}\lceil n) \backslash n_{0}\right)\right.\right.$.

Hence, applying $\left(\otimes^{4}\right)+\left(\otimes^{2}\right)+$ the definition of $k_{1}(-)$, we conclude that

$\left(\otimes^{5}\right) \quad\left(\forall n \geq n_{1}\right)\left(k_{1}\left(\bar{\alpha}^{1}\lceil n)=k_{1}\left(\bar{\alpha}^{2}\lceil n)\right)\right.\right.$,

and then applying $\left(\otimes^{4}\right)+\left(\otimes^{2}\right)+\left(\otimes^{5}\right)+$ the definition of $k_{0}(-)$, we get

$\left(\otimes^{6}\right) \quad$ for all $n \geq n_{1}$ : either $k_{0}\left(\bar{\alpha}^{1}\lceil n)=\max u\left(\bar{\alpha}^{1}\right)\right.$ and $k_{0}\left(\bar{\alpha}^{2}\lceil n)=\right.$ $\max u\left(\bar{\alpha}^{2}\right)$, or $k_{0}\left(\bar{\alpha}^{1}\lceil n)=k_{0}\left(\bar{\alpha}^{2}\lceil n)\right.\right.$.

Since

$$
\left(\forall n \geq n_{0}\right)\left(f_{n}\left(\bar{\alpha}^{1}\right)=f_{n}\left(\bar{\alpha}^{2}\right) \& g_{n}\left(\bar{\alpha}^{1}\right)=g_{n}\left(\bar{\alpha}^{2}\right)\right)
$$

and by $\left(\otimes^{2}\right)+\left(\otimes^{5}\right)+$ the choice of $n_{0}+$ the definition of $\mathbf{l}(-)$, we get (compare the proof of 2.6.1)

$\left(\otimes^{7}\right) \quad\left(\forall n \geq n_{1}\right)\left(\mathbf{l}\left(\bar{\alpha}^{1}\lceil n)=\mathbf{l}\left(\bar{\alpha}^{2}\lceil n)\right)\right.\right.$

and by $\left(\otimes^{2}\right)+\left(\otimes^{7}\right)+\left(\otimes^{6}\right)+$ the definition of $\mathbf{m}(-)$,

$$
\left(\forall n \geq n_{3}\right)\left(\mathbf { m } \left(\bar{\alpha}^{1}\lceil n)=\mathbf{m}\left(\bar{\alpha}^{2}\lceil n) \geq n_{2}\right) .\right.\right.
$$


Moreover, now we easily get

$$
\left(\forall n \geq n_{3}\right)\left(\mathbf { k } \left(\bar{\alpha}^{1}\lceil n)=\mathbf{k}\left(\bar{\alpha}^{2}\lceil n)\right) .\right.\right.
$$

For integers $n_{0} \leq n_{1} \leq n_{2}$ we define functions $F_{n_{0}, n_{1}, n_{2}}^{0}:{ }^{n_{2}} \lambda \rightarrow \mathcal{H}\left(\aleph_{0}\right)$ by letting $F_{n_{0}, n_{1}, n_{2}}^{0}\left(\alpha_{0}, \ldots, \alpha_{n_{2}-1}\right)$ (for $\left\langle\alpha_{0}, \ldots, \alpha_{n_{2}-1}\right\rangle \in n_{2} \lambda$ ) be the sequence consisting of:

(a) $\left\langle n_{0}, n_{1}, n_{2}\right\rangle$

(b) the set $T_{n_{1}, n_{2}}$ of all terms $\tau_{l}^{n}$ such that $n \leq n_{2}-n_{1}$ and either $l \leq n_{2}$ (we will call it the simple case) or $\tau_{l}^{n}$ is a composition of depth at most $n_{2}$ of such terms,

(c) $\left\langle\eta_{\alpha} \mid n_{2}, n, l,\left\langle i_{0}, \ldots, i_{n-1}\right\rangle\right\rangle$ for $n \leq n_{2}-n_{1}, i_{0}, \ldots, i_{n-1} \in\left[n_{1}, n_{2}\right)$ and $l$ such that $\tau_{l}^{n} \in T_{n_{1}, n_{2}}$ and $\alpha=\tau_{l}^{n}\left(\alpha_{i_{0}}, \ldots, \alpha_{i_{n-1}}\right)$,

(d) $\left\langle n, l,\left\langle i_{0}, \ldots, i_{n-1}\right\rangle, i\right\rangle$ for $n \leq n_{2}-n_{1}, i_{0}, \ldots, i_{n-1} \in\left[n_{1}, n_{2}\right), i \in$ $\left[n_{0}, n_{1}\right)$ and $l$ such that $\tau_{l}^{n} \in T_{n_{1}, n_{2}}$ and $\alpha_{i}=\tau_{l}^{n}\left(\alpha_{i_{0}}, \ldots, \alpha_{i_{n-1}}\right)$,

(e) equalities among appropriate terms, i.e. all tuples

$$
\left\langle n^{\prime}, l^{\prime}, n^{\prime \prime}, l^{\prime \prime},\left\langle i_{0}^{\prime}, \ldots, i_{n^{\prime}-1}^{\prime}\right\rangle,\left\langle i_{0}^{\prime \prime}, \ldots, i_{n^{\prime \prime}-1}^{\prime \prime}\right\rangle\right\rangle
$$

such that $n_{1} \leq i_{0}^{\prime}<\ldots<i_{n^{\prime}-1}^{\prime}<n_{2}, n_{1} \leq i_{0}^{\prime \prime}<\ldots<i_{n^{\prime \prime}-1}^{\prime \prime}<n_{2}$, $n^{\prime}, n^{\prime \prime} \leq n_{2}-n_{1}, l^{\prime}, l^{\prime \prime}$ are such that $\tau_{l^{\prime}}^{n^{\prime}}, \tau_{l^{\prime \prime}}^{n^{\prime \prime}} \in T_{n_{1}, n_{2}}$ and

$$
\tau_{l^{\prime}}^{n^{\prime}}\left(\alpha_{i_{0}^{\prime}}, \ldots, \alpha_{i_{n^{\prime}-1}^{\prime}}\right)=\tau_{l^{\prime \prime}}^{n^{\prime \prime}}\left(\alpha_{i_{0}^{\prime \prime}}, \ldots, \alpha_{i_{n^{\prime \prime}-1}^{\prime \prime}}\right) .
$$

(Note that the value of $F_{n_{0}, n_{1}, n_{2}}^{0}(\bar{\alpha})$ does not depend on $\bar{\alpha}\left\lceil n_{0}\right.$.)

Finally we define functions $F_{n}:{ }^{n} \lambda \rightarrow \mathcal{H}\left(\aleph_{0}\right)$ (for $\left.1<n<\omega\right)$ by:

$$
\text { if } \bar{\alpha} \in{ }^{n} \lambda \quad \text { then } \quad F_{n}(\bar{\alpha})=F_{\mathbf{k}(\bar{\alpha}), \mathbf{l}(\bar{\alpha}), n}^{0}(\bar{\alpha}) .
$$

As $\mathcal{H}\left(\aleph_{0}\right)$ is countable we may think that these functions are into $\omega$. We are going to show that they witness $\mathcal{K} \mathcal{L}(\lambda, \omega)$.

Claim 2.6.3. If $\bar{\alpha}^{1}, \bar{\alpha}^{2} \in{ }^{\omega} \lambda$ are such that $\left(\forall^{\infty} n\right)\left(\alpha_{n}^{1}=\alpha_{n}^{2}\right)$ then

$$
\left(\forall^{\infty} n\right)\left(F _ { n } \left(\bar{\alpha}^{1}\lceil n)=F_{n}\left(\bar{\alpha}^{2}\lceil n)\right) .\right.\right.
$$

Proof. Take $m_{0}<\omega$ such that for all $n \in\left[m_{0}, \omega\right)$ we have

$$
\alpha_{n}^{1}=\alpha_{n}^{2}, \quad \mathbf{l}\left(\bar{\alpha}^{1}\lceil n)=\mathbf{l}\left(\bar{\alpha}^{2}\lceil n), \quad \mathbf{k}\left(\bar{\alpha}^{1}\lceil n)=\mathbf{k}\left(\bar{\alpha}^{2}\lceil n)\right.\right.\right.\right.
$$

(possible by 2.6.2). Let $m_{1}>m_{0}$ be such that for all $n \geq m_{1}$,

$$
\mathbf{k}\left(\bar{\alpha}^{1}\lceil n)=\mathbf{k}\left(\bar{\alpha}^{2}\lceil n)>m_{0}\right.\right.
$$

(use 2.6.1). Then, for $n \geq m_{1}, i=1,2$ we have

$$
F_{n}\left(\bar{\alpha}^{i}\lceil n)=F_{\mathbf{k}\left(\bar{\alpha}^{i}\lceil n), \mathbf{l}\left(\bar{\alpha}^{i}\lceil n), n\right.\right.}^{0}\left(\bar{\alpha}^{i}\lceil n)=F_{\mathbf{k}\left(\bar{\alpha}^{1}\lceil n), \mathbf{l}\left(\bar{\alpha}^{1}\lceil n), n\right.\right.}^{0}\left(\bar{\alpha}^{i}\lceil n) .\right.\right.\right.
$$

Since the value of $F_{n_{0}, n_{1}, n_{2}}^{0}(\bar{\beta})$ does not depend on $\bar{\beta}\left\lceil n_{0}\right.$ and the sequences $\bar{\alpha}^{1}\left\lceil n, \bar{\alpha}^{2}\left\lceil n\right.\right.$ agree on $\left[m_{0}, \omega\right)$, we get

$$
F_{\mathbf{k}\left(\bar{\alpha}^{1}\lceil n), \mathbf{l}\left(\bar{\alpha}^{1}\lceil n), n\right.\right.}^{0}\left(\bar{\alpha}^{1}\lceil n)=F_{\mathbf{k}\left(\bar{\alpha}^{1}\lceil n), \mathbf{l}\left(\bar{\alpha}^{1}\lceil n), n\right.\right.}^{0}\left(\bar{\alpha}^{2}\lceil n)=F_{\mathbf{k}\left(\bar{\alpha}^{2}\lceil n), \mathbf{l}\left(\bar{\alpha}^{2}\lceil n), n\right.\right.}^{0}\left(\bar{\alpha}^{2}\lceil n),\right.\right.\right.
$$


and hence

$$
\left(\forall n \geq m_{1}\right)\left(F _ { n } \left(\bar{\alpha}^{1}\lceil n)=F_{n}\left(\bar{\alpha}^{2}\lceil n)\right) .\right.\right.
$$

Claim 2.6.4. If $\bar{\alpha}^{1}, \bar{\alpha}^{2} \in{ }^{\omega} \lambda$ and $\left(\forall^{\infty} n\right)\left(F_{n}\left(\bar{\alpha}^{1}\lceil n)=F_{n}\left(\bar{\alpha}^{2}\lceil n)\right)\right.\right.$ then $\left(\forall^{\infty} n\right)\left(\alpha_{n}^{1}=\alpha_{n}^{2}\right)$

Proof. Take $n_{0}<\omega$ such that

$$
u\left(\bar{\alpha}^{1}\right) \cup u\left(\bar{\alpha}^{2}\right) \subseteq n_{0} \quad \text { and } \quad\left(\forall n \geq n_{0}\right)\left(F _ { n } \left(\bar{\alpha}^{1}\lceil n)=F_{n}\left(\bar{\alpha}^{2}\lceil n)\right) .\right.\right.
$$

Then for all $n \geq n_{0}$ we have (by clause (a) of the definition of $F_{n_{0}, n_{1}, n_{2}}^{0}$ )

$$
\mathbf{l}\left(\bar{\alpha}^{1}\lceil n)=\mathbf{l}\left(\overline { \alpha } ^ { 2 } \lceil n ) \text { and } \mathbf { k } \left(\bar{\alpha}^{1}\lceil n)=\mathbf{k}\left(\bar{\alpha}^{2}\lceil n) .\right.\right.\right.\right.
$$

Further, let $n_{1}>n_{0}$ be such that for all $n \geq n_{1}, \mathbf{k}\left(\bar{\alpha}^{1}\lceil n)>n_{0}\right.$ and $k_{0}\left(\bar{\alpha}^{1}\left\lceil n_{1}\right)=\max u\left(\bar{\alpha}^{1}\right)\right.$ (exists by 2.6.1) and choose $n_{2}>n_{1}$ such that $n \geq n_{2}$ implies $\mathbf{m}\left(\bar{\alpha}^{1}\lceil n)>n_{2}\right.$.

We are going to show that $\alpha_{n}^{1}=\alpha_{n}^{2}$ for all $n>n_{1}$. Assume not. Then we have $n>n_{1}$ with $\alpha_{n}^{1} \neq \alpha_{n}^{2}$ and thus $\eta_{\alpha_{n}^{1}} \neq \eta_{\alpha_{n}^{2}}$. Take $n^{\prime}>n$ such that $\eta_{\alpha_{n}^{1}}\left\lceil n^{\prime} \neq \eta_{\alpha_{n}^{2}}\left\lceil n^{\prime}\right.\right.$. Applying 2.6.1(2) and (4) choose $n^{\prime \prime}>n^{\prime}$ such that

$$
\mathbf{m}\left(\overline { \alpha } ^ { 1 } \lceil n ^ { \prime \prime } ) > n ^ { \prime } \quad \text { and } \quad k _ { 0 } \left(\bar{\alpha}^{1}\left\lceil n^{\prime \prime}\right)=\max u\left(\bar{\alpha}^{1}\right) .\right.\right.
$$

Now define inductively: $m_{0}=n^{\prime \prime}, m_{k+1}=\mathbf{m}\left(\bar{\alpha}^{1}\left\lceil m_{k}\right)\right.$. Thus

$$
n^{\prime \prime}=m_{0}>\mathbf{l}\left(\bar{\alpha}^{1}\left\lceil m_{0}\right) \geq m_{1}>\mathbf{l}\left(\bar{\alpha}^{1}\left\lceil m_{1}\right) \geq m_{2}>\ldots\right.\right.
$$

and (by induction on $k$ )

$$
m_{k}>\max u\left(\bar{\alpha}^{1}\right) \Rightarrow k_{0}\left(\bar{\alpha}^{1}\left\lceil m_{k}\right)=\max u\left(\bar{\alpha}^{1}\right)\right.
$$

(see the definition of $\mathbf{m}$ ). Let $k^{*}$ be the first such that $n \geq m_{k^{*}}$ (so $k^{*} \geq 2$, exists by the choice of $n_{1}$ ). Note that by the choice of $n_{1}$ above we necessarily have

$$
m_{k^{*}}>\mathbf{l}\left(\bar{\alpha}^{1}\left\lceil m_{k^{*}}\right)=\mathbf{k}\left(\bar{\alpha}^{1}\left\lceil m_{k^{*}-1}\right)>n_{0} .\right.\right.
$$

Hence for all $k<k^{*}$ :

$$
\begin{gathered}
F_{m_{k}}\left(\bar{\alpha}^{1}\left\lceil m_{k}\right)=F_{m_{k}}\left(\bar{\alpha}^{2}\left\lceil m_{k}\right),\right.\right. \\
\mathbf{l}\left(\bar{\alpha}^{1}\left\lceil m_{k+1}\right)=\mathbf{l}\left(\bar{\alpha}^{2}\left\lceil m_{k+1}\right)=\mathbf{k}\left(\bar{\alpha}^{1}\left\lceil m_{k}\right)=\mathbf{k}\left(\bar{\alpha}^{2}\left\lceil m_{k}\right) .\right.\right.\right.\right.
\end{gathered}
$$

By the definition of the functions $\mathbf{l}, \mathbf{m}, \mathbf{k}$ and the choice of $m_{0}$ (remember $k_{0}\left(\bar{\alpha}^{1}\left\lceil m_{0}\right)=\max u\left(\bar{\alpha}^{1}\right)\right)$ we know that for each $i \in\left[\mathbf{k}\left(\bar{\alpha}^{1}\left\lceil m_{k}\right), \mathbf{l}\left(\bar{\alpha}^{1}\left\lceil m_{k}\right)\right)\right.\right.$ and $k<k^{*}$, for some $\tau_{l}^{m} \in T_{\mathbf{l}\left(\bar{\alpha}^{1}\left\lceil m_{k}\right), m_{k}\right.}$ and $i_{0}, \ldots, i_{m-1} \in\left[\mathbf{l}\left(\bar{\alpha}^{1}\left\lceil m_{k}\right), m_{k}\right)\right.$ we have $\alpha_{i}^{1}=\tau_{l}^{m}\left(\alpha_{i_{0}}^{1}, \ldots, \alpha_{i_{m-1}}^{1}\right)$. Moreover we may demand that $\tau_{l}^{m}$ is a composition of depth at most $\mathbf{l}\left(\bar{\alpha}^{1} \mid m_{k}\right)-i$ of simple case terms. Since

$$
F_{\mathbf{k}\left(\bar{\alpha}^{1}\left\lceil m_{k}\right), \mathbf{l}\left(\bar{\alpha}^{1}\left\lceil m_{k}\right), m_{k}\right.\right.}^{0}\left(\bar{\alpha}^{1}\left\lceil m_{k}\right)=F_{\mathbf{k}\left(\bar{\alpha}^{2}\left\lceil m_{k}\right), \mathbf{l}\left(\bar{\alpha}^{2}\left\lceil m_{k}\right), m_{k}\right.\right.}^{0}\left(\bar{\alpha}^{2}\left\lceil m_{k}\right)\right.\right.
$$

we conclude that (by clause (d) of the definition of the functions $F_{n_{0}, n_{1}, n_{2}}^{0}$ )

$$
\alpha_{i}^{2}=\tau_{l}^{m}\left(\alpha_{i_{0}}^{2}, \ldots, \alpha_{i_{m-1}}^{2}\right) .
$$


Now look at our $n$. If $\mathbf{l}\left(\bar{\alpha}^{1}\left\lceil m_{k^{*}-1}\right)>n\right.$ then $\mathbf{k}\left(\bar{\alpha}^{1}\left\lceil m_{k^{*}-1}\right) \leq n<\right.$ $\mathbf{l}\left(\bar{\alpha}^{1}\left\lceil m_{k^{*}-1}\right)\right.$ and thus we find $i_{0}, \ldots, i_{m-1} \in\left[\mathbf{l}\left(\bar{\alpha}^{1}\left\lceil m_{k^{*}-1}\right), m_{k^{*}-1}\right)\right.$ and $\tau_{l}^{m} \in$ $T_{\mathbf{1}\left(\bar{\alpha}^{1} \mid m_{k^{*}-1}\right), m_{k^{*}-1}}$ such that

$$
\alpha_{n}^{1}=\tau_{l}^{m}\left(\alpha_{i_{0}}^{1}, \ldots, \alpha_{m-1}^{1}\right) \quad \text { and } \quad \alpha_{n}^{2}=\tau_{l}^{m}\left(\alpha_{i_{0}}^{2}, \ldots, \alpha_{m-1}^{2}\right) .
$$

If $\mathbf{l}\left(\bar{\alpha}^{1}\left\lceil m_{k^{*}-1}\right) \leq n\right.$ then $n \in\left[\mathbf{k}\left(\bar{\alpha}^{1}\left\lceil m_{k^{*}-2}\right), \mathbf{l}\left(\bar{\alpha}^{1}\left\lceil m_{k^{*}-2}\right)\right)\left(\operatorname{as~} \mathbf{l}\left(\bar{\alpha}^{1}\left\lceil m_{k^{*}-1}\right)\right.\right.\right.\right.$ $=\mathbf{k}\left(\bar{\alpha}^{1}\left\lceil m_{k^{*}-2}\right)\right.$ and $n<m_{k^{*}-1} \leq \mathbf{l}\left(\bar{\alpha}^{1}\left\lceil m_{k^{*}-2}\right)\right)$. Hence, for some $i_{0}, \ldots, i_{m-1}$ $\in\left[\mathbf{l}\left(\bar{\alpha}^{1}\left\lceil m_{k^{*}-2}\right), m_{k^{*}-2}\right)\right.$ and $\tau_{l}^{m} \in T_{\mathbf{l}\left(\bar{\alpha}^{1} \mid m_{k^{*}-2}\right), m_{k^{*}-2}}$, we have

$$
\alpha_{n}^{1}=\tau_{l}^{m}\left(\alpha_{i_{0}}^{1}, \ldots, \alpha_{m-1}^{1}\right) \quad \text { and } \quad \alpha_{n}^{2}=\tau_{l}^{m}\left(\alpha_{i_{0}}^{2}, \ldots, \alpha_{m-1}^{2}\right) .
$$

In both cases we may additionally demand that the term $\tau_{l}^{m}$ is a composition of depth $\mathbf{l}\left(\bar{\alpha}^{1}\left\lceil m_{k^{*}-1}\right)-n\right.$ (or $\mathbf{l}\left(\bar{\alpha}^{1}\left\lceil m_{k^{*}-2}\right)-n\right.$, respectively) of terms of the simple case. Now we proceed inductively (taking care of the depth of the terms involved) and we find a term $\tau \in T_{\mathbf{l}\left(\bar{\alpha}^{1} \mid m_{0}\right), m_{0}}$ (which is a composition of depth at most $\mathbf{l}\left(\bar{\alpha}^{1}\left\lceil m_{0}\right)-n\right.$ of terms of the simple case) and $i_{0}, \ldots, i_{m-1} \in\left[\mathbf{l}\left(\bar{\alpha}^{1}\left\lceil m_{0}\right), m_{0}\right)\right.$ such that

$$
\alpha_{n}^{1}=\tau\left(\alpha_{i_{0}}^{1}, \ldots, \alpha_{m-1}^{1}\right) \quad \text { and } \quad \alpha_{n}^{2}=\tau\left(\alpha_{i_{0}}^{2}, \ldots, \alpha_{m-1}^{2}\right) .
$$

But now applying clause (c) of the definition of the functions $F_{n_{0}, n_{1}, n_{2}}^{0}$ we conclude that $\eta_{\alpha_{n}^{1}}\left\lceil m_{0}=\eta_{\alpha_{n}^{2}}\left\lceil m_{0}\right.\right.$, contradicting the choice of $n^{\prime}$ and the fact that $m_{0}>n^{\prime}$

The last two claims finish the proof of the theorem.

REMARK 2.7. If the model $M$ has $\kappa<\lambda$ functions (so $\left\langle\tau_{i}^{n}\left(x_{0}, \ldots, x_{n-1}\right)\right.$ : $i<\kappa\rangle$ lists the $n$-place terms) we can prove $\mathcal{K} \mathcal{L}(\lambda, \kappa)$ and the proof is similar.

Final Remarks 2.8. (1) Now we phrase exactly what is needed to carry out the proof of Theorem 1.1 for $\lambda>\kappa$. It is:

( $\otimes) \quad$ for every model $M$ with universe $\lambda$ and Skolem functions and with countable vocabulary, we can find pairwise distinct $\alpha_{n, l}<\lambda$ (for $n<\omega, l<\omega)$ such that

$(\otimes) \quad$ if $m_{0}<m_{1}<\omega$ and $l_{i}^{\prime}<l_{i}^{\prime \prime}$ for $i<m_{0}$ and $l_{i}<\omega$ for $i \in\left[m_{0}, m_{1}\right)$ and $k_{0}<k_{1}<k_{2}<\omega$ then the models

$$
\begin{gathered}
\left(\operatorname{Sk}\left(\left\{\alpha_{i, l_{i}^{\prime}}, \alpha_{i, l_{i}^{\prime \prime}}: i<m_{0}\right\} \cup\left\{\alpha_{m_{0}, k_{0}}, \alpha_{m_{0}, k_{1}}\right\} \cup\left\{\alpha_{i, l_{i}}: i \in\left(m_{0}, m_{1}\right)\right\}\right),\right. \\
\alpha_{0, l_{0}^{\prime}}, \alpha_{0, l_{0}^{\prime \prime}}, \alpha_{1, l_{1}^{\prime}}, \alpha_{1, l_{1}^{\prime \prime}}, \ldots, \alpha_{m_{0}-1, l_{m_{0}-1}^{\prime}}, \alpha_{m_{0}-1, l_{m_{0}-1}^{\prime \prime}}, \alpha_{m_{0}, k_{0}}, \\
\left.\alpha_{m_{0}, k_{1}}, \alpha_{m_{0}+1, l_{m_{0}+1}}, \ldots, \alpha_{m_{1}-1, l_{m_{1}-1}}\right)
\end{gathered}
$$

and

$$
\begin{gathered}
\left(\operatorname{Sk}\left(\left\{\alpha_{i, l_{i}^{\prime}}, \alpha_{i, l_{i}^{\prime \prime}}: i<m_{0}\right\} \cup\left\{\alpha_{m_{0}, k_{0}}, \alpha_{m_{0}, k_{2}}\right\} \cup\left\{\alpha_{i, l_{i}}: i \in\left(m_{0}, m_{1}\right)\right\}\right),\right. \\
\alpha_{0, l_{0}^{\prime}}, \alpha_{0, l_{0}^{\prime \prime}}, \alpha_{1, l_{1}^{\prime}}, \alpha_{1, l_{1}^{\prime \prime}}, \ldots, \alpha_{m_{0}-1, l_{m_{0}-1}^{\prime}}, \alpha_{m_{0}-1, l_{m_{0}-1}^{\prime \prime}}, \alpha_{m_{0}, k_{0}}, \\
\left.\quad \alpha_{m_{0}, k_{2}}, \alpha_{m_{0}+1, l_{m_{0}+1}}, \ldots, \alpha_{m_{1}-1, l_{m_{1}-1}}\right)
\end{gathered}
$$


are isomorphic and the isomorphism is the identity on their intersection and they have the same intersection with $\kappa$.

For more details and more related results we refer the reader to [Sh:F254].

(2) Together with 1.5, 2.7 this gives a good bound on the consistency strength of $\neg \mathcal{K} \mathcal{L}(\lambda, \kappa)$.

(3) What if we ask $F_{n}:{ }^{n} \lambda \rightarrow{ }^{\omega>} \kappa$ such that $F_{n}(\eta) \unlhd F_{n+1}(\eta)$ and $\eta \in{ }^{\omega} \lambda \Rightarrow F(\eta)=\bigcup F_{n}\left(\eta\lceil n) \in{ }^{\omega} \kappa\right.$ ? No real change.

\section{References}

[Ka90] S. Kalikow, Sequences of reals to sequences of zeros and ones, Proc. Amer. Math. Soc. 108 (1990), 833-837.

[Ko84] P. Koepke, The consistency strength of the free-subset property for $\omega_{\omega}$, J. Symbolic Logic 49 (1984), 1198-1204.

[Mi91] A. W. Miller, Arnie Miller's problem list, in: H. Judah (ed.), Set Theory of the Reals (Ramat Gan, 1991), Israel Math. Conf. Proc. 6, Bar-Ilan Univ., Ramat Gan, 1993, 645-654.

[Sh 76] S. Shelah, Independence of strong partition relation for small cardinals, and the free-subset problem, J. Symbolic Logic 45 (1980), 505-509.

[Sh 124] —, א $\aleph_{\omega}$ may have a strong partition relation, Israel J. Math. 38 (1981), 283288 .

[Sh 110] - Better quasi-orders for uncountable cardinals, ibid. 42 (1982), 177-226.

[Sh:b] —, Proper Forcing, Lecture Notes in Math. 940, Springer, Berlin, 1982.

[Sh:g] —, Cardinal Arithmetic, Oxford Logic Guides 29, Oxford Univ. Press, 1994.

[Sh 481] -, Was Sierpiński right? III Can continuum-c.c. times c.c.c. be continuumc.c.? Ann. Pure Appl. Logic 78 (1996), 259-269.

[Sh:F254] - More on Kalikow Property of pairs of cardinals.

[Sh 513] - PCF and infinite free subsets, Arch. Math. Logic, to appear.

[Si70] J. Silver, A large cardinal in the constructible universe, Fund. Math. 69 (1970), 93-100.

Institute of Mathematics

The Hebrew University of Jerusalem 91904 Jerusalem, Israel

E-mail: shelah@math.huji.ac.il
Department of Mathematics Rutgers University New Brunswick, NJ 08854, U.S.A. URL: http://www.math.rutgers.edu/ shelah 